\title{
Evaluación de la efectividad, seguridad y costos del tratamiento antimicrobiano intravenoso ambulatorio (TAIA) vs hospitalizado en infección urinaria en pediatría
}

\author{
Anamaría Peña, Alexsandra Zambrano, Muriel Alvarado, Jaime Cerda y Rodrigo Vergara
}

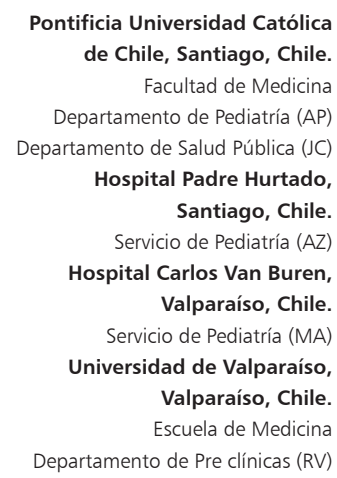

Lugar donde se efectuó el estudio Servicio de Pediatría del Complejo

Asistencial Dr. Sótero del Río Servicio de Pediatría del Hospital Carlos van Buren.

Los autores declaran no tener conflictos de interés Financiamiento: No hubo Este trabajo correspondió a la tesis para optar al título de Magister en Ciencias Médicas con mención en Infecciones Intrahospitalarias y Epidemiología Hospitalaria de la Universidad de Valparaíso de Anamaría Peña Donati

Recibido: 29 de enero de 2013 Aceptado: 4 de julio de 2013

Correspondencia a: Rodrigo Vergara Fisher rodrigo.vergara@uv.c

\section{Introducción}

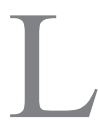
a hospitalización de un niño para tratamiento antimicrobiano intravenoso conlleva riesgos para el paciente (siendo el más importante adquirir una infección asociada a la atención de salud [IAAS]), genera alteraciones de la dinámica familiar (trastornos emocionales, ausencia laboral y licencias médicas de las madres, ausencia a centros de cuidado y gastos por traslados), y además significa un costo económico importante para el sistema de salud público.

En contraposición a este modelo tradicional, surge la atención ambulatoria para tratamiento parenteral, que permite mantener al paciente en su entorno familiar y administrar el tratamiento antimicrobiano intravenoso (IV) mientras sea necesario. Este modelo denominado Tratamiento antimicrobiano intravenoso ambulatorioTAIA (en inglés Outpatient parenteral antimicrobial therapy- OPAT $)^{1}$, ha sido ampliamente utilizado en otros países, en patologías específicas de pacientes mayoritariamente adultos ${ }^{2-18}$, otorgando atenciones seguras para el paciente, a un menor costo y logrando la satisfacción del usuario ${ }^{1}$.

La evaluación de costos realizada en países desarrollados, demuestra que TAIA es entre 3 y 6 veces más barato que el tratamiento hospitalizado, siendo el principal factor de ahorro los días-cama utilizados ${ }^{19-22}$.

En Pediatría, la experiencia con TAIA ha sido reducida; sin embargo, la disponibilidad de antimicrobianos de uso parenteral de amplio espectro y de vida media prolongada, ha permitido su uso en pacientes con determinadas enfermedades que cuenten con tutores responsables, con fácil acceso y comunicación con el hospital ${ }^{1,4,23,24}$. Un estudio prospectivo en niños entre 3 meses y 5 años con infección urinaria (ITU) febril demostró una efectividad de 96,6\% y adherencia al tratamiento en $87,1 \%{ }^{6}$.

En la mayoría de los centros hospitalarios chilenos los niños son hospitalizados para tratamiento antimicrobiano IV. Sin embargo, desde el año 2003, el Complejo Asistencial Dr. Sótero del Río (CADSR), cuenta con una unidad de Hospitalización Transitoria (UHT) para tratamiento intravenoso ambulatorio de niños beneficiarios del 
Servicio de Salud Metropolitano Sur-Oriente (SSMSO) y las prestaciones realizadas allí han evitado numerosas hospitalizaciones, lo que se ha traducido en mayor disponibilidad de camas y ahorro económico para el servicio ${ }^{23}$.

Considerando que la ITU es motivo frecuente de visita en nuestros servicios de urgencia pediátricos $(1,35 \%$ del total de consultas) y que cerca de $10 \%$ deriva en hospitalización ${ }^{25}$, se planificó este estudio para comparar la efectividad, seguridad y costos del tratamiento IV hospitalizado versus TAIA en niños con ITU febril.

\section{Pacientes y Métodos}

Estudio analítico, observacional, de cohorte concurrente, en dos cohortes dinámicas de niños con ITU febril atendidos en dos hospitales públicos tipo 1, de alta complejidad: una ambulatoria (TAIA) tratada en la UHT pediátrica del CADSR de la Región Metropolitana de Santiago y otra hospitalizada en el Servicio de Pediatría (SP) del Hospital Carlos van Buren (HCVB) de la V Región.

\section{Selección de los participantes}

Los pacientes con fiebre y sospecha de ITU planteada por el médico tratante (considerando la presentación clínica y el resultado de sedimento de orina), consultantes en las Unidades de Emergencia Infantil (UEIs) de ambos servicios fueron invitados a participar en el estudio, para lo cual sus padres firmaron un consentimiento informado. Los criterios de inclusión fueron: niños de 2 meses a 5 años con indicación de antimicrobiano IV, estabilidad hemodinámica, urocultivo tomado por cateterismo vesical previo al tratamiento, madre o tutor legal alfabetos. Para la cohorte TAIA se exigió fácil acceso al hospital y disponibilidad de teléfono fijo o móvil. Se excluyeron los pacientes con indicación de hospitalización en unidades críticas, fracaso de tratamiento antimicrobiano oral o intramuscular previo, deshidratación al ingreso, co-morbilidad, antecedente de patología nefro-urológica, inmunosupresión o patología crónica. Fueron eliminados aquellos con urocultivo de ingreso negativo o contaminado o no encontrado.

En ambos centros el sedimento de orina se efectuó con muestra centrifugada y observación por campo y la muestra de orina se cultivó en medios agar sangre y agar McConkey. Se consideró confirmada la ITU si hubo crecimiento de un microorganismo con recuento mayor a $10^{4} \mathrm{ufc} / \mathrm{ml}$ en urocultivo tomado por cateterismo transuretral, negativo si no hubo crecimiento bacteriano, $\mathrm{y}$ contaminado si hubo crecimiento de un microorganismo que normalmente coloniza la piel o los genitales con recuento menor o igual a $10^{4} \mathrm{ufc} / \mathrm{ml}$ o si hubo crecimiento de dos o más microorganismos en el urocultivo tomado por cateterismo vesical.

Los pacientes seleccionados en la UEI del CADSR fueron derivados a la UHT para evaluación médica diaria hasta su alta y continuación de tratamiento antimicrobiano IV cada 24 horas hasta efectuar cambio a terapia vía oral. Aquellos hospitalizados en el SP del HCVB fueron tratados de acuerdo a la normativa de ese servicio. En ambos centros los pacientes fueron tratados de la manera habitual para cada uno de ellos. Las únicas intervenciones que contempló el protocolo fueron el registro de exámenes, insumos y medicamentos gastados, prestaciones del equipo de salud y la encuesta a los padres sobre gastos y tiempos perdidos (días laborales, asistencia a centros de cuidado). En ambas cohortes se programó un último control después del alta (mínimo tres días después de terminado el tratamiento antimicrobiano). Los inasistentes fueron controlados por teléfono.

\section{Evaluación de la efectividad del tratamiento}

Se midió con el resultado del urocultivo posterior a la suspensión de los antimicrobianos, considerando tratamiento efectivo (curación) si no hubo crecimiento del microorganismo que causó la ITU. Se comparó el porcentaje de niños curados en ambas cohortes.

\section{Evaluación de efectos adversos y seguridad}

Durante todo el período que duró el estudio, ambos equipos efectuaron una pesquisa activa de re-hospitalizaciones (cohorte del HCVB) y de hospitalizaciones (cohorte del CADSR) atribuibles a la atención de salud efectuada. Los eventos adversos (EAs) derivados de la vía venosa, del antimicrobiano y las IAAS fueron pesquisados mientras estuvieron en control, en el control posterior al alta y por llamada telefónica a los pacientes ausentes. En el grupo hospitalizado también se revisó la ficha clínica. La seguridad se comparó a través de las tasas de los EAs.

\section{Estimación de costos directos e indirectos}

Para el costo directo se calculó el costo día-cama de pediatría, día-cama integral ambulatoria pediátrica, exámenes de laboratorio, antimicrobianos, insumos, otros medicamentos utilizados y prestaciones del equipo de salud (médicos, enfermera, técnico para-médico y otros profesionales). Los costos de día-cama y día-cama integral ambulatoria fueron extraídos del programa $\mathrm{WinSIG}^{26,27} \mathrm{de}$ ambos centros. Los costos de exámenes de laboratorio y prestaciones del equipo de salud fueron extraídos del Arancel FONASA (Fondo Nacional de Salud, sistema de seguro Estatal) Modalidad Institucional, año $2011^{28}$ y los de antimicrobianos, otros medicamentos e insumos, del portal Chile compra de enero 2011.

El costo indirecto se midió por pérdida de días laborales, días de inasistencia a sala cuna, jardín infantil o colegio y gastos económicos efectuados por los padres (compra de medicamentos no disponibles en los centros, alimentación y traslados al centro hospitalario). 
Todos los costos monetarios fueron expresados en moneda nacional y dólar americano (valor al 31 de enero de $2011^{29}$ ).

Se calculó el costo promedio en ambos grupos (costo directo promedio más costo indirecto promedio) y se efectuó la comparación mediante una razón. Adicionalmente se calculó el índice costo-curación del episodio de ITU en ambos centros considerando el total de pacientes curados y descontando las pérdidas de seguimiento y aquellos no curados.

Se midieron las pérdidas de seguimiento en ambas cohortes, el porcentaje de fracaso y de adherencia a TAIA (porcentaje de asistencia a la UHT para tratamiento IV de acuerdo a las citaciones médicas).

La recolección de los datos fue efectuada por el equipo médico y enfermeras de cada centro (previamente entrenados), en forma directa y prospectiva, mediante instrumentos con instructivos y definiciones, y validados en un período de marcha blanca. Los datos se analizaron en el programa Epi Info versión 3.4.1.

Se efectuó análisis descriptivo con medidas de concentración y dispersión para las variables cuantitativas y comparación de las características de ambas poblaciones por análisis univariado. Las pruebas de significación estadística usadas fueron: PDH para proporciones independientes validada por prueba exacta de Fisher, PDH no paramétrica validada con test Mann Whitney, PDH para medias de grupos independientes con la prueba $t$ de Student. Se calcularon tasas, RR e IC95\%, pruebas de $\chi^{2}$ y se consideró nivel de significación estadística el valor p: 0,05 .

El estudio fue aprobado por los Comités de Evaluación Ético-Científico del SSMSO y del Servicio de Salud Valparaíso-San Antonio (SSVSA).

\section{Resultados}

En el período noviembre 2009-noviembre 2010, 238 niños con diagnóstico de ITU febril, fueron tratados con TAIA en la UHT del CADSR, siendo la mala tolerancia oral la indicación de tratamiento IV en $100 \%$ de ellos; 118 niños fueron excluidos y de 120 incluidos nueve fueron eliminados. El análisis se efectuó en 111 pacientes enrolados (cohorte TAIA). Entre enero de 2010 y junio de 2011, 216 niños fueron hospitalizados en el SP del HCVB. Ciento cuatro niños fueron excluidos y de 112 incluidos, 31 fueron eliminados. El análisis se efectuó en 81 pacientes enrolados (cohorte hospitalizada). Hubo una pérdida de seguimiento de 7,3\%: 14 pacientes: $6(5,4 \%)$ de TAIA y ocho hospitalizados (9,9\%) El análisis de los costos y seguridad se hizo en 192 pacientes: 111 de la cohorte TAIA y 81 de la hospitalizada, y el de efectividad se efectuó en 178: 105 y 73, respectivamente (Figura 1).
Las características demográficas se describen en la Tabla 1. Ochenta y seis $(77,5 \%)$ de la cohorte TAIA y $48(59,3 \%)$ de la hospitalizada eran mujeres (p: 0,01). El promedio de edad fue 14 meses $\pm 11,6$ en la cohorte TAIA y 10 meses $\pm 10,7$ en la hospitalizada (p: 0,07 ) y la moda ocho y tres meses, respectivamente. La mayor parte de los niños de ambas cohortes residía en zonas urbanas y no hubo diferencia en la existencia de trabajo remunerado de las madres ni en la proporción de niños que asistía a centros de cuidado.

Los 111 niños ingresados al estudio para tratamiento con TAIA completaron el tratamiento IV mientras se mantuvo la indicación médica logrando una adherencia a TAIA de $100 \%$. Ninguno fue hospitalizado como consecuencia del fracaso de TAIA.

Los antimicrobianos IV utilizados fueron amikacina, ceftriaxona y cefotaxima. En la cohorte TAIA se usó mayoritariamente amikacina $(87,4 \%)$ y como alternativa ceftriaxona $(12,6 \%)$ cuando el diagnóstico inicial fue bacteriemia oculta. En nueve niños $(8,1 \%)$ se cambió el antimicrobiano IV (por persistencia de fiebre o por confirmación del diagnóstico de ITU). En la cohorte hospitalizada el antimicrobiano más usado fue cefotaxima $(97.5 \%)$ y menos frecuente amikacina $(2,5 \%)$. En nueve casos $(11,1 \%)$ se cambió el antimicrobiano inicial (por mala respuesta clínica o ajuste según antibiograma). El tratamiento antimicrobiano IV duró en promedio 3,2 $\pm 4,1$ días (rango 1-6) en la cohorte TAIA y 5,1 \pm 2 días (rango 3-12) en la hospitalizada.

\section{Evaluación de la efectividad del tratamiento}

Se efectuó con los pacientes controlados con urocultivo posterior al término del tratamiento. En quienes no se hizo este urocultivo se efectuó contacto telefónico (seis de TAIA y uno de la cohorte hospitalizada) y control clínico en cuatro de este grupo, encontrándose todos asintomáticos.

El urocultivo se tomó en promedio 3,9 $\pm 1,3$ días después de terminado el tratamiento antimicrobiano en la cohorte TAIA y 7,7 \pm 8,3 días en la hospitalizada. En este grupo no fue posible cumplir con el plazo programado por problemas administrativos, y por norma interna se tomó la muestra por recolector de orina, a diferencia del grupo ambulatorio en el que se hizo por cateterismo vesical.

En la cohorte TAIA, el urocultivo fue negativo en $102(97,1 \%)$ y hubo contaminación de la muestra en tres $(2,9 \%)$, en todos resultó negativo al repetirlo, de tal forma que la efectividad del tratamiento fue $100 \%$. En la cohorte hospitalizada, 66 de los urocultivos fueron negativos $(90,4 \%)$, cuatro tuvieron crecimiento bacteriano $(8,2 \%)$ y tres resultaron contaminados $(4,1 \%)$, uno de ellos fue negativo al repetirlo. Un paciente con urocultivo positivo fue hospitalizado por un nuevo episodio de ITU febril causado por el mismo microorganismo y el estudio demos- 


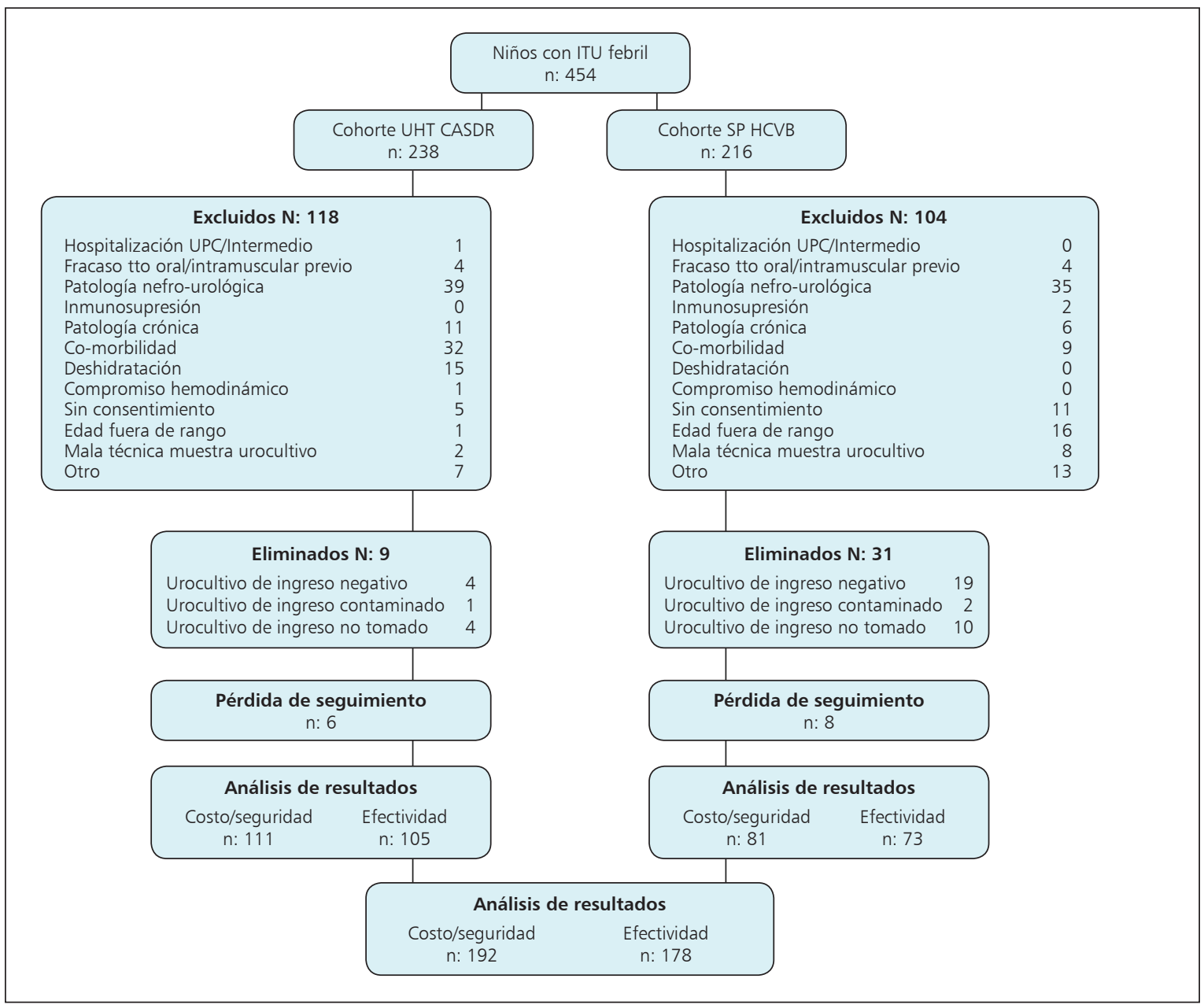

Figura 1. Flujograma de enrolamiento de pacientes.

tró que tenía hidro-ureteronefrosis. En los tres restantes el microorganismo diagnosticado fue distinto al que causó la ITU que motivó el ingreso al estudio diagnosticándose un nuevo episodio y se consideró curado el primero. Hubo curación en 72 niños de la cohorte hospitalizada, alcanzando a 98,6\% de efectividad del tratamiento. La comparación de la efectividad de ambos tratamientos no demostró diferencia (RR: 0,99, IC95\% 0,96-1,01).

\section{Evaluación de eventos adversos y seguridad}

La tasa de EAs en la cohorte hospitalizada fue 71,6\% (58 pacientes) comparada con $16,2 \%$ en TAIA (18 pacientes); RR: 4,42 (IC95\% 2,83-6,89). Para el grupo hospitalizado se obtuvo un riesgo atribuible de 55,4 y una fracción atribuible de $77,4 \%$.

Los 58 pacientes del grupo hospitalizado tuvieron 104 episodios de EAs (1,8 eventos por paciente); 17 tuvieron dos EAs, nueve tuvieron tres, en dos hubo cuatro y en uno ocurrieron 5 EAs.
Tabla 1. Características demográficas de ambas cohortes de niños ( 2 meses-5 años) con ITU febril

\begin{tabular}{lccc|} 
Características demográficas & $\begin{array}{c}\text { Cohorte TAIA* } \\
(\mathbf{n}: \mathbf{1 1 1})\end{array}$ & $\begin{array}{c}\text { Cohorte hospitalizada } \\
(\mathbf{n}: \mathbf{8 1})\end{array}$ & valor $\mathbf{p}$ \\
Género femenino & $86(77,5 \%)$ & $48(59,3 \%)$ & 0,01 \\
Edad (meses) & $14 \pm 11,6$ & $10 \pm 10,7$ & 0,07 \\
Promedio \pm DS & $(2-50)$ & $(2-60)$ & \\
(rango) & $106(95,5 \%)$ & $78(96,2 \%)$ & 0,54 \\
Residencia & $5(4,5 \%)$ & $3(3,8 \%)$ & 0,74 \\
Urbana & $32(28,8 \%)$ & $26(32,1 \%)$ & 0,79 \\
Rural & $22(19,8 \%)$ & $14(17,3 \%)$ & \\
Trabajo materno remunerado & 12 & 10 & \\
Asistencia a centro de cuidado & 6 & 4 & \\
Sala cuna & 4 & 0 & \\
Jardín infantil & & & \\
Colegio & & & \\
*TAlA: terapia antimicrobiana intravenosa ambulatoria. & & \\
\hline
\end{tabular}


En la cohorte TAIA hubo sólo 18 EAs en 18 pacientes.

La Tabla 2 muestra las tasas específicas de EAs. El $94,8 \%$ de los pacientes hospitalizados tuvo EAs de vía venosa, $8,6 \%$ presentó IAAS y $6,9 \%$ del antimicrobiano, sólo un paciente tuvo uno derivado del tratamiento y correspondió a una madre que quedó atrapada en un ascensor, En la cohorte TAIA la mitad de los pacientes con EAs tuvo uno relacionado a vía venosa. La cohorte hospitalizada tuvo una tasa específica de EAs de vía venosa significativamente más alta $(67,9 \%$ comparada con TAIA $[8,1 \%], \mathrm{RR}=8,37$ (IC95\% 4,4-15,94) no se encontraron diferencias entre las tasas de EAs derivados del antimicrobiano y de IAAS.

\begin{tabular}{|c|c|c|c|c|c|c|}
\hline \multirow[t]{2}{*}{ Evento adverso } & \multicolumn{2}{|c|}{$\begin{array}{c}\text { Cohorte TAIA* } \\
\text { n: } 111\end{array}$} & \multicolumn{2}{|c|}{$\begin{array}{l}\text { Cohorte hospitalizada } \\
\text { n: } 81\end{array}$} & \multirow[t]{2}{*}{ RR } & \multirow[t]{2}{*}{ IC95\% } \\
\hline & $\mathbf{n}$ & Tasa x 100 & $\mathrm{n}$ & Tasa x 100 & & \\
\hline Vía venosa & 9 & 8,1 & 55 & 67,9 & 8,37 & $4,4-15,94$ \\
\hline Antimicrobiano & 5 & 4,5 & 4 & 4,9 & 1,10 & $0,30-3,96$ \\
\hline IAAS & 4 & 3,6 & 5 & 6,2 & 1,71 & $0,47-6,18$ \\
\hline
\end{tabular}

\begin{tabular}{|c|c|c|}
\hline Evento adverso & $\begin{array}{c}\text { Cohorte TAIA* } \\
n\end{array}$ & $\begin{array}{c}\text { Cohorte hospitalizada } \\
\text { n }\end{array}$ \\
\hline Vía venosa & 9 & 94 \\
\hline Obstrucción & 4 & 20 \\
\hline Salida accidental & 4 & 36 \\
\hline Extravasación & 1 & 37 \\
\hline Flebitis & 0 & 1 \\
\hline Antimicrobiano & 5 & 4 \\
\hline Vómitos & 2 & 0 \\
\hline Diarrea & 2 & 3 \\
\hline Rash & 1 & 1 \\
\hline IAAS & 4 & 5 \\
\hline IRA viral & 2 & 1 \\
\hline IRA virus parainfluenza 3 & 1 & 0 \\
\hline IRA por adenovirus & 1 & 1 \\
\hline Diarrea por rotavirus & 0 & $3^{*}$ \\
\hline Tratamiento & 0 & 1 \\
\hline Accidente del paciente & 0 & 0 \\
\hline Accidente de padres & 0 & 1 \\
\hline Total & 18 & 104 \\
\hline
\end{tabular}

En la Tabla 3 se muestran los tipos de EAs. En ninguna de las dos cohortes hubo letalidad por IAAS, pero derivaron en prolongación de la hospitalización, re-hospitalización, gasto adicional por exámenes de laboratorio y aumento del gasto de los padres.

\section{Estimación de costos}

El índice costo-curación calculado en los 177 pacientes curados fue 3,9 veces superior en el grupo hospitalizado. Curar a un niño entre 2 meses y 5 años con ITU costó \$ 707.278 en el SP del HCVB comparado con $\$ 182.697$ en la UHT del CADSR.

Costos directos: La cohorte TAIA fue controlada 419 días (promedio 3,8 \pm 1 días por paciente) y fue tratada con TAIA, 333 días (promedio $3 \pm 1$ día por paciente, rango 1-6; mediana y moda 3). La cohorte hospitalizada permaneció 435 días internada (promedio 5,7 $\pm 1,8$ días). La diferencia promedio de días TAIA versus días de hospitalización fue 2,7 días ( $\mathrm{p}<0,05)$.

El costo día-cama ambulatoria para la UHT del CADSR fue $\$ 31.073$ (promedio de los costos del primer semestre de $2011^{25}$ ) y el día-cama Pediatría del HCVB fue $\$ 94.086$ (promedio de los costos de enero y mayo de 2011, meses en que se aplicó el programa WinSIG ${ }^{26}$ ).

La Tabla 4 muestra los costos directos de atención. El costo promedio día-cama representó más de $3 / 5$ del costo directo total en ambas cohortes. El costo promedio de TAIA correspondió a 21,8\% del costo de la cohorte hospitalizada.

Las prestaciones del equipo de salud representaron $1 / 5$ del costo directo total y fueron 3,3 veces superiores en el grupo hospitalizado. El mayor número de atenciones, en ambas cohortes, correspondió a las efectuadas por técnico paramédico; sin embargo, las atenciones médicas representaron el mayor costo.

Los exámenes de laboratorio representaron un poco menos de 1/5 del costo directo total y su costo fue 2,5 veces superior en la cohorte hospitalizada.

La valorización de los antimicrobianos orales, otros medicamentos (antipiréticos, soluciones parenterales y medición de electrolitos plasmáticos) e insumos usados representó un costo marginal.

El costo directo promedio en la cohorte hospitalizada fue muy superior al de TAIA (\$696.790, US\$1.443 versus $\$ 170.928$, US\$354) $(p<0,01)$. Para verificar diferencias de costo entre los pacientes con estadías más prolongadas y mayor consumo de antimicrobianos, insumos y exámenes de laboratorio, se calculó el costo directo de la mediana encontrándose una diferencia no significativa con el promedio en ambas cohortes y manteniéndose la relación 1:4 a favor de TAIA $(\mathrm{p}<0,01)$.

Costos indirectos: Se describen en la Tabla 5. En la cohorte TAIA, 20 de $32(62,5 \%)$ madres con trabajo remunerado se ausentaron de su trabajo en promedio 
2,7 días, sólo un tercio accedió a licencia médica. En la cohorte hospitalizada, tres estaban con licencia médica por otro motivo y 22 de $23(95,7 \%)$ madres que se encontraban trabajando se ausentaron en promedio 7,6 días (p: 0.01). En ambas cohortes un pequeño porcentaje accedió a licencia médica sin diferencia en el promedio de días (p: 0.11). Hubo diferencia en el promedio de días de inasistencia a centro de cuidado (4 en TAIA y 6,9 en hospitalizado, p: 0,045).

Aunque el gasto promedio total efectuado por los padres fue mayor en la cohorte hospitalizada, esta diferencia no fue significativa (p. 0,07). Sin embargo, se encontraron diferencias en algunos gastos específicos: el gasto en alimentación fue mayor en la cohorte hospitalizada y en la cohorte TAIA hubo más gasto en medicamentos.

Costo asociado a IAAS: En la cohorte TAIA sólo ocasionaron exceso de costo por exámenes de laboratorio, mientras que en la cohorte hospitalizada un paciente con diarrea por rotavirus debió ser re-hospitalizado para tratamiento y estudio, y en los otros se efectuaron exámenes de laboratorio. El mayor costo estuvo representado por el día-cama, $\$ 106.550$ en el grupo hospitalizado y $\$ 33.630$ en TAIA. El costo directo promedio en los pacientes con IAAS fue \$ 999.876 (US\$2.070) en la cohorte hospitalizada con un exceso de costo de $\$ 303.086$ (US\$628) y $\$ 230.910$ (US\$ 478) en la cohorte TAIA con un exceso de costo de $\$ 59.982$ (US\$124) ( $<<0,01)$.

\section{Discusión}

La hospitalización para tratamiento antimicrobiano IV constituye una indicación médica tradicional. Como alternativa a ella, hace más de tres décadas, surgió TAIA para el tratamiento de pacientes adultos con patologías y condiciones definidas, llegando a constituir una intervención innovadora con beneficios demostrados para los usuarios y establecimientos de salud ${ }^{30-32}$. La experiencia en niños es reducida, y casi exclusiva de países en desarrollo y su aplicación desde hace nueve años en Chile, específicamente en la UHT del CADSR, es inusual.

Este estudio evaluó indicadores económicos y de salud en pacientes atendidos en dos hospitales públicos de similares características. El análisis de homogeneidad de ambas cohortes demostró similitud en todas las características demográficas, excepto género; no obstante, no hay evidencias que permitan suponer que esta diferencia pueda influir en los resultados. Pese a que no hubo diferencia estadísticamente significativa en la edad, el grupo hospitalizado era de menor edad, lo que podría explicar la mayor tendencia a su hospitalización y la duración de ésta.

Los escasos estudios de TAIA en niños con ITU revelan una efectividad superior a $96 \%$, a $100 \%$, confirmando la hipótesis planteada. El uso de

\begin{tabular}{|c|c|c|}
\hline Costo & $\begin{array}{c}\text { Cohorte TAIA } \\
\text { (\$) }\end{array}$ & $\begin{array}{c}\text { Cohorte hospitalizada } \\
\text { (\$) }\end{array}$ \\
\hline Día-cama & 117.574 & 538.519 \\
\hline Equipo de salud & 33.482 & 112.852 \\
\hline Antimicrobianos & 2.346 & 4.766 \\
\hline Otros medicamentos & 1.031 & 1.306 \\
\hline Insumos & 1.420 & 2.033 \\
\hline Exámenes & 15.095 & 37.354 \\
\hline Costo directo promedio & $170.928^{*}$ & $696.790 * *$ \\
\hline Costo directo mediana & 171.930 & 645.956 \\
\hline
\end{tabular}

Tabla 5. Costos indirectos efectuados en niños ( 2 meses-5 años) con ITU febril de ambas cohortes

\begin{tabular}{|c|c|c|c|}
\hline Ausentismo laboral & $\begin{array}{l}\text { Cohorte TAIA } \\
\text { Madres n: } 32\end{array}$ & $\begin{array}{l}\text { Cohorte hospitalizada } \\
\text { Madres n: } 23\end{array}$ & valor $p$ \\
\hline $\mathrm{n}$ madres inasistentes & $20(62,5 \%)$ & $22(95,7 \%)$ & 0,01 \\
\hline $\mathrm{n}$ días inasistencia & 84 & 168 & \\
\hline Promedio días inasistencia & 4,2 & 7,6 & 0,01 \\
\hline Licencia médica & $10(31,3 \%)$ & $5(23,8 \%)$ & \\
\hline n días licencia médica & 51 & 58 & \\
\hline Promedio días licencia médica & 5,1 & 11,6 & 0,11 \\
\hline Ausentismo a centros de cuidado & Niños n: 22 & Niños n: 14 & \\
\hline n días inasistencia & 88 & 97 & \\
\hline Promedio días inasistencia & 4 & 6,9 & 0,07 \\
\hline Gastos monetarios & $\$$ & $\$$ & \\
\hline Alimentación & 2.317 & 5.604 & $<0,01$ \\
\hline Medicamentos & 1.340 & 339 & $<0,01$ \\
\hline Traslados & 9.047 & 9.332 & 0,81 \\
\hline Gasto promedio & 12.704 & 15.275 & 0,07 \\
\hline Gasto mediana & 12.000 & 13.800 & 0,07 \\
\hline
\end{tabular}

antimicrobianos IV de amplio espectro efectivos contra microorganismos causantes de ITU, con farmacocinética y farmacodinamia conocidas, contribuyó al éxito del resultado pese a la diferencia en los antimicrobianos IV usados en ambos centros.

La seguridad es un aspecto relevante de la atención de salud y en TAIA se describen EAs principalmente relacionados con accesos venosos centrales ${ }^{33,34}$; sin embargo, en este estudio se utilizó vía venosa periférica. El riesgo de presentar un EA fue 4,7 veces más alto en el grupo hospitalizado comparado con TAIA. Del análisis del riesgo atribuible se desprende que si los pacientes hospitalizados fuesen tratados con TAIA, 55 de cada 100 niños dejarían 
de tener EAs. De acuerdo a la fracción atribuible obtenida, se reduciría en 77,4\% los EAs en los niños hospitalizados si se trataran con TAIA. La ocurrencia de EAs de vía venosa fue 4,3 veces superior en la cohorte hospitalizada, con 1,6 episodios por cada niño con EAs, probablemente por la mayor manipulación del acceso venoso durante la estadía hospitalaria debido al fraccionamiento del antimicrobiano usado; la literatura médica confirma la escasa incidencia de estos EAs en TAIA ${ }^{6}$.

Uno de los potenciales beneficios de TAIA es el menor riesgo de IAAS ${ }^{32-35}$. Aunque en este estudio no se encontró una diferencia significativa en la tasa de IAAS, esto podría deberse a que el número de sujetos en la cohorte hospitalizada fue insuficiente para alcanzar el nivel de significancia. Probablemente el plazo de seguimiento puede haber sido también insuficiente en ambas cohortes; y en la cohorte hospitalizada el control posterior a su alta fue efectuado por los tratantes habituales, lo que podría haber disminuido la pesquisa de IAAS en relación a la cohorte TAIA cuyo seguimiento fue realizado por personal del estudio. A pesar de lo señalado, en la cohorte hospitalizada hubo tres casos de diarrea por rotavirus y ninguno en TAIA. Esta es una infección nosocomial potencialmente grave que causa prolongación de la hospitalización y aumento del costo ${ }^{36}$; un caso debió ser re-hospitalizado produciendo exceso de costo por día-cama y exámenes de laboratorio. En ambos grupos hubo un caso de infección por adenovirus, agente causante de infecciones pulmonares graves con posibles secuelas e incluso muerte ${ }^{37,38}$; ambos pacientes tuvieron buena evolución, registrándose exceso de costo por exámenes de laboratorio.

El significativo menor costo de TAIA comparado con el tratamiento hospitalizado, es uno de los beneficios más relevantes al momento de escoger una intervención cuando la efectividad y la seguridad están aseguradas ${ }^{1,14,19-22}$. En este estudio, el costo de TAIA representó un cuarto del costo de la atención hospitalizado. El mayor costo estuvo representado por el día-cama y en menor proporción por las prestaciones del equipo de salud; el conjunto restante (medicamentos, insumos y exámenes de laboratorio), significó un costo marginal.

La evaluación de un programa TAIA demuestra un significativo ahorro económico para los centros hospitala$\operatorname{rios}^{39}$. En el período estudiado se internaron 136 pacientes por año en el SP del HCVB por la misma patología, su tratamiento con TAIA habría significado un importante ahorro para este servicio (\$71.517.232, US\$147.977).

La mayoría de las publicaciones incluye el costo directo de atención y muy pocas analizan el costo indirecto y generalmente está referido a la pérdida de días laborales. En este estudio se intentó estimar varios de los costos indirectos, para lo cual se incluyó la pérdida de días laborales de las madres y de asistencia a centros de cuidado de los niños. No fue posible calcular el costo monetario ya que en la encuesta no se incluyó el sueldo de las madres por considerarse confidencial. Hubo mayor pérdida de días laborales en la cohorte hospitalizada por la permanencia de las madres junto a sus hijos durante la hospitalización. Se incluyó los gastos de los padres por traslados al hospital, alimentación y compra de medicamentos. Una publicación ${ }^{20}$ concluyó el mayor gasto de los pacientes con TAIA; sin embargo, en nuestro estudio no se encontró diferencia en el gasto promedio total. El mayor gasto por alimentación en la cohorte hospitalizada se puede explicar por la mayor permanencia de los padres en el recinto. En la cohorte TAIA hubo mayor gasto por compra de medicamentos; si bien esto fue un costo marginal, podría solucionarse con la entrega de todos los medicamentos por parte del centro asistencial. No es posible descartar errores en la estimación de costos por traslados cuando éstos se efectuaron en vehículo particular y los padres calcularon el gasto en combustible.

Una variable no evaluada en este estudio fue la satisfacción del usuario. Datos no publicados revelan un alto nivel de aceptación en la UHT del CADSR. La adherencia de $100 \%$ al seguimiento en TAIA apoya indirectamente una alta satisfacción.

La imposibilidad de efectuar este estudio en un centro que cuente con las dos modalidades de tratamiento IV (ambulatoria y hospitalizada) para esta patología, no permitió realizar la comparación en condiciones igualitarias, lo que ciertamente es una limitación. Si bien las poblaciones atendidas en los dos hospitales no son iguales, ambas son fundamentalmente beneficiarias y de acuerdo al censo del año $2002^{40}$ son similares en cuanto a nivel de ruralidad, alfabetización y pobreza. Por otra parte, es posible que existan diferencias en las conductas clínicas de los médicos, de manera que eventualmente un paciente hospitalizado por ITU en el CADSR pudiera tener un promedio menor de días hospitalizado y diferencias en otras acciones que generen menores costos que los que se obtuvieron en el HCVB. Si bien esto puede afectar la magnitud de las diferencias de costos obtenidos en este estudio, no afectaría el resultado general, que verifica una clara disminución de costos en los pacientes tratados ambulatoriamente. Después de la realización de este estudio y considerando sus resultados, se implementó exitosamente la modalidad de TAIA en el HCVB. Otra limitación de este estudio que pudiera tener mayor importancia, es la diferencia de edades en los dos grupos comparados. Si bien ésta no alcanzó significancia estadística, el grupo hospitalizado tuvo en promedio cuatro meses menos que la cohorte TAIA. Es posible que esta diferencia sea explicada por una mayor tendencia de los médicos del HCVB por indicar hospitalización a los niños más pequeños y tratamiento oral a algunos de los mayores ante la imposibilidad de optar por tratamiento IV ambulatorio. También fueron diferentes los antimicrobianos utilizados 
en ambos hospitales de acuerdo a su normativa. Sin embargo, es difícil que estas diferencias puedan afectar de manera importante los resultados del estudio, ya que no se ha descrito que ellas afecten la eficacia del tratamiento de una ITU ${ }^{41-43}$. Si bien no se realizó el urocultivo de control de seguimiento en $100 \%$ de los sujetos, 7 de los 9 niños sin control fueron contactados telefónicamente, encontrándose asintomáticos. Es improbable que esta pérdida de control haya influido en los resultados. El método de toma de muestra de urocultivo de control fue distinto en los dos sitios, pero esto no afectaría sustancialmente los resultados. El principal problema de la toma de muestra por bolsa recolectora es que tiene una baja especificidad ${ }^{43}$, lo que podría haber disminuido la efectividad de la modalidad de tratamiento hospitalizada. Esto no parece haber ocurrido, ya que la efectividad fue cercana a $100 \%$ en los dos centros.

En conclusión, TAIA en ITU en niños entre 2 meses y 5 años fue igualmente efectivo, más seguro en términos de EAs derivados de la vía venosa y significativamente menos costoso que el tratamiento hospitalizado, lo que la transforma en una intervención beneficiosa, tanto para los pacientes como para el sistema de salud público, favorable en cuanto a minimización de costo y desde el punto de vista de satisfacción del usuario, por lo que sería recomendable implementarlo en hospitales o servicios pediátricos del sistema público, similares a los estudiados.

Agradecimientos. Al equipo de la UHT del CADSR, especialmente a Alejandra Prado y becarios de Pediatría e Infectología de la Pontificia Universidad Católica de Chile y Universidad de Valparaíso que hicieron posible la realización del estudio, y las enfermeras Cristina Gajardo y Emelina
Veillón y técnico paramédico Carmen Soto, que efectuaron los registros de insumos y medicamentos.

\section{Resumen}

Introducción: La hospitalización de niños para tratamiento antimicrobiano intravenoso, ha sido reemplazada en países desarrollados y en algunos centros chilenos por el tratamiento antimicrobiano intravenoso ambulatorio (TAIA). Objetivo: Comparar efectividad, seguridad y costos de TAIA versus hospitalizado. Pacientes y Métodos: Se efectuó un estudio de cohorte prospectiva en niños (2 meses-5 años) con infección urinaria (ITU) febril atendidos en dos hospitales públicos chilenos: una cohorte ambulatoria y otra hospitalizada. Entre noviembre 2009 y 2010, se enrolaron 111 niños en TAIA y entre enero 2010-junio 2011, 81 niños hospitalizados. Se registraron datos demográficos, gastos de atención y de los padres, respuesta al tratamiento, eventos adversos y complicaciones. Resultados: No hubo diferencia en la efectividad de ambos tratamientos (100\% en TAIA y $98,6 \%$ en hospitalizado; p: 0,41$)$. La adherencia a TAIA fue $100 \%$. La prevalencia de eventos adversos fue mayor en los hospitalizados (76,3 versus $16,2 \%, \mathrm{p}<0,01)$. El costo directo promedio fue cuatro veces superior en hospitalizados, principalmente por costo día-cama. El costo indirecto fue similar. Hubo más días de ausentismo laboral y a centros de cuidado en hospitalizados (p: 0,017, p: 0,045, respectivamente). Conclusión: El tratamiento de ITU febril con TAIA en niños fue igualmente efectivo, más seguro y significativamente menos costoso que el hospitalizado y representa una intervención recomendable para los servicios pediátricos de hospitales públicos chilenos.

\section{Referencias bibliográficas}

1.- Tice A, Rehm S, Dalovisio J, Bradley J, Martinelli L, Graham D el al. Practice guidelines for outpatient parenteral antimicrobial therapy. Clin Infect Dis 2004; 38: $1651-72$

2.- Bernard L, El-Hajj, Pron B, Lotthé A, Gleizes V, Signoret F et al. Outpatient parenteral antimicrobial therapy (OPAT) for the treatment of osteomyelitis: evaluation of efficacy, tolerance and cost. J Clin Pharm Ther 2001; 26 (6): 445-51

3.- Mauceri A. Treatment of bone and joint infections utilizing a third-generation cephalosporin with an outpatient drug delivery device. Am J Med 1994; 97: 14-22.

4.- Bradley J, Ching D, Phillips S. Outpatient therapy of serious pediatric infections with ceftriaxone. Pediatr Infect Dis J 1988; 7 : 160-4.
5.- Morales J O, Snead H. Efficacy and safety of intravenous cefotaxime for treating pneumonia in outpatients. Am J Med 1994; 97: 28-33.

6.- Gauthier M, Chevalier I, Sterescu A, Bergeron S, Brunet S and Taddeo D. Treatment of urinary tract infections among febrile young children with daily intravenous antibiotic therapy at a day treatment center. Pediatrics 2004; 114 (4): 469-76

7.- Miller L K, Wing D A, Paul R H, Grimes D A. Outpatient treatment of pyelonephritis in pregnancy: a randomized controlled trial. Obstet Gynecol 1995; 86: 560-4.

8.- Seaton R, Bell E, Gourlay Y, Semple L. Nurse-led management of uncomplicated cellulitis in the community: evaluation of a protocol incorporating intravenous ceftriaxone. J Antimicrob Chemother 2005; 55 (5): 764-7.

9.- Nathwani D. The management of skin and soft tissue infections: outpatient parenteral antibiotic therapy in the United Kingdom. Chemotherapy 2001; 47: 17-23.

10.- Poretz D. Treatment of skin and soft-tissue infections utilizing an outpatient parenteral drug delivery device: A multicenter trial. Am J Med 1994; 97: 23-7.

11.- Waler J, Rathore M. Outpatient management of bacterial meningitis. Pediatr Infect Dis J 1995; 14 (2): 89-93.

12.- Stamboulian D. Outpatient treatment of endocarditis in a clinic-based program in Argentina. Eur J Clin Microbiol Infect Dis 1995; 14 (7): 648-54 Review.

13.- Francioli P, Etienne J, Hoigne R, Thys J, Gerber A. Treatment of streptococcal endocarditis with a single daily dose of ceftriaxone sodium for 4 weeks: efficacy and outpatient treatment feasibility. JAMA 1992; 267: 264-7.

14.- Mullen C, Petropoulos D, Roberts W M, 
Rytting M, Zipf T, Chan K, et al. Economic and resource utilization analysis of outpatient management of fever and neutropenia in lowrisk pediatric patients with cancer.

J Ped Hematol Oncol 1999; 21: 212-8.

15.- Mullen C, Petropoulos D, Roberts M, Rytting M, ZipfT, Chan K, et al. Outpatient treatment of fever and neutropenia for low risk pediatric cancer patients. Cancer 1999; 86: 126-34.

16.- Mustafa M, Aquino, Pappo A, Tkaczewski I, Buchanan G. A pilot study of outpatient management of febrile neutropenic children with cancer at low risk of bacteremia. J Pediatr 1996; 128: 847-9.

17.- Balaguer A. and González J. Intravenous antibiotics given at home for people with cystic fibrosis. The Cochrane Database of Systematic Reviews 2009 Issue 1.

18.- Morales J, Von Behren L. Secondary bacterial infections in HIV infected patients: an alternative ambulatory outpatient treatment utilizing intravenous cefotaxime. Am J Med 1994; 97: 9-13.

19.- Wai A, Frighetto L, Marra C, Chan E, Jewesson P. Cost analysis of an adult outpatient parenteral antibiotic therapy (OPAT) programme. A Canadian teaching hospital and Ministry of Health perspective. Pharmacoeconomics 2000; 18 (5): 451-7.

20.- Eisenberg J, Kitz D. Savings from outpatient antibiotic for osteomyeltis: economic analysis of a therapeutic strategy. JAMA 1986; 255 : 1584-8.

21.- Tice A. Pharmacoeconomic considerations in the ambulatory use of parenteral cephalosporins. Drugs 2000; 59 Suppl 3: 29-35

22.- Nguyen M, Hoze R. Hospital Based OPAT at a University Hospital. Abstracts from 44th Annual Meeting of, October 12-5, 2006, Toronto

23.- Peña A, Viviani T, Le Corre N, Morales V, Montecinos C. Manejo de la infección del tracto urinario en lactantes febriles: Experiencia de tratamiento antimicrobiano intravenoso ambulatorio. Rev Chilena Infectol 2009; 26(4): 350-4.

24.- Bartal C, Danon A, Schlaeffer F, Reisenberg K, Alkan M, Smoliakov R, et al. Pharmacokinetic dosing of aminoglycosides: a controlled trial. Am J Med 2003; 114 (3): 194-8.

25.- Lizama M, Luco M, Reichhard C, Hirsh T. Infección del tracto urinario en un servicio de urgencia pediátrico: frecuencia y características clínicas. Rev Chilena Infectol 2005; 22: 235-41.

26.- Reporte Departamento de Finanzas Complejo Asistencial Dr. Sótero del Río, I Semestre año 2011. Programa de costeo WinSIG

27.- Reporte Departamento de Finanzas Hospital Carlos Van Buren, año 2011. Programa de costeo WinSIG.

28.- Arancel FONASA Modalidad Institucional, año 2011.

29.- Base de datos económicos del Banco Central de Chile, valor dólar al 31 de Enero de 2011. http://si2.bcentral.cl/ Basededatoseconomicos/951_480.asp. (accedido el 30 de marzo de 2011).

30.- Leggett J. Ambulatory use of parenteral antibacterials. contemporary perspectives. Drugs 2000; 59 Suppl 3: S1-S8.

31.- Chapman A, Seaton A, Cooper M, Hedderwick S, Goodall V, Reed C, et al. Good practice recommendations for outpatient parenteral antimicrobial therapy (OPAT) in adults in the UK: a consensus statement. J Antimicrob Chemother 2012; 67: 1053-62.

32.- Paladino J, Poretz D. Outpatient antimicrobial therapy today. Clin Infect Dis 2010; 51 (Suppl 2): S198-S208.

33.- Gómez M, Maraga N, Alvarez A, Rathore M. Complications of outpatient parenteral antibiotic therapy in childhood. Pediatr Infect Dis J 2001; 20 (5): 541-3.

34.- Rathore $M$. The unique issues of outpatient parenteral antimicrobial therapy in children and adolescents. Clin Infect Dis 2010; 51 (Suppl 2): S209-15.

35.- Tice A and Rehm S. Meeting the challenges of methicillin-resistant Staphylococcus aureus with outpatient parenteral antimicrobial therapy. Clin Infect Dis 2010; 51 (Suppl 2): S171-5.

36.- Delpiano L, Riquelme J, Casado M, Álvarez X. Comportamiento clínico y costos de la gastroenteritis por rotavirus en lactantes: Adquisición comunitaria versus nosocomial. Rev Chilena Infectol 2006; 23 (1): 35-42.

37.- Palomino $M$, Larrañaga $C$, Avendaño L. Hospital-acquired adenovirus $7 \mathrm{~h}$ infantile respiratory infection in Chile. Pediatr Infect Dis J 2000; 19: 527-31.

38.- Pérez M J, Kogan R, Maggi L, Mendoza C. Seguimiento clínico y factores de riesgo en niños con enfermedades respiratorias por adenovirus. Rev Chil Pediatr 2007; 78 (3): 261-7.

39.- Nguyen H. Hospitalist to home: outpatient parenteral antimicrobial therapy at an academic center. Clin Infect Dis 2010; 51 (Suppl 2): S220-3.

40.- Base de datos del Censo 2002, INE. http://www. ine.cl/cd2002/sintesiscensal.pdf (accedido el 12 de junio de 2013).

41.- Salas del C. P, Barrera P, González C, Zambrano P, Salgado I, Quiroz L, et al. Actualización en el diagnóstico y manejo de la infección urinaria en pediatría. Rev Chil Pediatr 2012; 83 (3): 269-78.

42.- White B. Diagnosis and treatment of urinary tract infections in children. Am Fam Physician 2011; 83 (4): 409-15.

43.- American Academy of Pediatrics. Urinary Tract Infection: Clinical practice guideline for the diagnosis and management of the initial UTI in febrile infants and children 2 to 24 months, Subcommittee on Urinary Tract Infection and Steering Committee on Quality Improvement and Management. Pediatrics 2011; 128 (3): 595-610. 